\title{
Revolutionary Advances in Immunotherapy for Melanoma Are Coming into the Surgical Arena: Are We Ready?
}

\author{
Charles M. Balch, MD, FACS, FASCO \\ University of Texas MD Anderson Cancer Center, Houston, TX
}

In the past decade, powerful new immunotherapy agents have demonstrated remarkable improvements in survival of patients with many histological types of stage IV cancer, as first demonstrated in metastatic melanoma and reported in 2010. ${ }^{1}$ With a new understanding of the immune system and a growing portfolio of powerful immunotherapy agents, survival rates in stage IV melanoma patients have dramatically increased from a historical median survival of 2-9 months, to a remarkable median survival exceeding 24 months, with approximately $20 \%$ of patients remaining in remission after 8 years. ${ }^{2-5}$

I predict that immunotherapy will radically transform the treatment of stage II and III melanoma over the next 5-10 years, with similar results in other cancers as well. This will involve expansion of the role of surgery in multidisciplinary management of stage IV cancers to include removal of residual tumors, as well as performance of molecular testing of refractory metastatic cancer cells after exposure to systemic therapy. As surgical oncologists, we must be knowledgeable about and engaged in incorporating these effective new systemic treatments into our multidisciplinary treatment planning and must enroll patients in clinical trials using checkpoint inhibitors and other new forms of immunotherapy.

The "breakthrough" biological concept that led to these dramatic clinical advances was the demonstration that metastatic human cancer cells can "blindfold" the patient's immune system, allowing metastases to proliferate unchecked until they destroy vital organ function. ${ }^{4}$ This concept explains why an array of immune stimulants and

\section{(C) Society of Surgical Oncology 2018}

First Received: 1 May 2018;

Published Online: 11 May 2018

C. M. Balch, MD, FACS, FASCO

e-mail: cmbalch@mdanderson.org vaccines have failed in the past decades. Thus, in a "tolerant state," most cancer patients cannot respond to treatment approaches that involve increased antigen presentation (e.g., vaccines) or immune stimulants (e.g., interferons or interleukins). With the availability of checkpoint inhibitors to "break tolerance," the immune system can now recognize and destroy metastases as it would any "foreign invader," such as viruses or transplanted tissues. With multiple checkpoint inhibitors approved for cancer, an entirely new landscape of systemic treatments is now being tested in cancer clinical trials. These include various combinations and sequences of checkpoint inhibitors and immune-stimulating agents to fully engage the immune system. Also, such strategies will increasingly include combinations of immunotherapies that activate the immune system and traditional systemic agents (targeted therapies, molecular therapies, and cytotoxic chemotherapy) that directly destroy metastases.

The indications and endpoints for evaluating new systemic agents in the adjuvant/neoadjuvant setting are somewhat different than those used in stage IV cancer trials, where the goal is palliation and the endpoint is usually improved survival in a population in which all patients usually eventually die from distant metastases. In contrast, trials that evaluate use of systemic agents before or after cancer surgery are intended to delay or eliminate occult distant metastases and improve overall survival. In this setting, we have to be careful about selecting patients who are most likely to benefit from systemic therapies because of the increased risk of occult distant metastases, and not unduly expose "good prognosis" patients to these new agents, as they would not gain any benefit from the systemic treatment being tested yet would unnecessarily bear the toxicity and cost.

This Educational Series on Immunotherapy for Cancers reviews the current status of adjuvant and neoadjuvant immunotherapy in melanoma, focusing on the future role 
of checkpoint inhibitors and a "first-of-its-kind" genetically engineered oncolytic virus. It includes four articles, focusing on (1) adjuvant immunotherapy for melanoma, ${ }^{6}$ (2) clinical trials for neoadjuvant immunotherapy for different cancers, ${ }^{7}$ (3) how to safely implement the genetically engineered oncolytic virus talimogene laherparepvec (TVEC) as a novel form of immunotherapy, ${ }^{8}$ and (4) longterm survival of patients with surgically resectable stage IV melanoma in a randomized immunotherapy trial. ${ }^{9}$

Drs. Bello and Ariyan from the Memorial Sloan Kettering Cancer Center provide a nice overview of adjuvant immunotherapy trials for stage III and resected stage IV melanoma and stress the importance of demonstrating a true survival benefit that exceeds toxicity and cost, based on evidence from properly designed and analyzed clinical trials. ${ }^{6}$ Dr. Keung and colleagues from the University of Texas MD Anderson Cancer Center describe the current state of neoadjuvant checkpoint inhibitor trials for a range of human cancers. ${ }^{7}$ They properly emphasize that we must be cautious in the design of these trials to avoid extended periods of systemic therapy administration, which might result in patients losing the opportunity to undergo successful surgical resection, especially if they have to endure prolonged recovery from a serious immunological complication of the experimental therapy. These authors also emphasize that pathological complete response (pCR) rates may not always translate into improved survival, as has been observed in some patients with breast cancer following pCR to neoadjuvant systemic therapy. On the other hand, neoadjuvant approaches are probably the most definitive way to determine whether any systemic treatment has been successful based upon pathological examination of the tumor mass and assessment of the components of the mass to distinguish between chronic inflammation/scarring and viable tumor tissue.

The third article provides an update about a novel, firstof-its-kind agent, talimogene laherparepvec (T-VEC), an intralesional oncolytic virus, in melanoma. ${ }^{8}$ T-VEC represents a significant feat in genetic engineering and drug development in several ways, as it was produced via manipulation of a herpes simplex virus as follows: (1) deletion of the gene responsible for its disease symptoms, rendering it nontoxic, (2) insertion of a gene that directs the virus to "home" to and infect melanoma tumor cells, and (3) insertion of a gene that directs tumor cells bearing the virus to produce large quantities of interferon (IFN)- $\gamma$ that can elicit a strong inflammatory response to destroy tumor cells in the surrounding area as well as those at remote sites. ${ }^{10},{ }^{11}$ This novel approach to cancer therapy is a forerunner to use of other genetically engineered viruses as vectors for delivery of cancer agents directly into primary tumors and their metastases.
These remarkable and sophisticated clinical investigations of T-VEC were led by Dr. Robert Andtbacka, a surgical oncologist from the University of Utah. His pioneering leadership exemplifies the ability of surgical oncologists to conduct drug development clinical trials that can lead to Food and Drug Administration (FDA) approval and be adopted into our patient care armamentarium. T-VEC is indicated for local treatment of unresectable cutaneous, subcutaneous, and nodal melanoma lesions in patients whose disease recurs following initial surgery. ${ }^{12}$ As discussed in Dr. Andtbacka's article, recent data suggest that T-VEC induces responses in both injected and uninjected lesions ${ }^{11}$ and that it can be used in combination with checkpoint inhibitors. ${ }^{13}$ Studies have also suggested that T-VEC may play a role in changing the tumor microenvironment, thereby enhancing the effects of checkpoint inhibitors. In a recent phase $1 \mathrm{~b}$ study, T-VEC plus the antiprogrammed death (PD-1) agent pembrolizumab yielded a $62 \%$ objective response rate in patients with unresectable stage IIIB-IV melanoma. ${ }^{14}$ Patients who responded demonstrated increased $\mathrm{CD} 8^{+} \mathrm{T}$ cell infiltration, elevated PD-L1 protein expression, and IFN- $\gamma$ gene expression in injected lesions. These findings indicate that T-VEC may alter the tumor microenvironment in a manner that improves the efficacy of anti-PD-1 therapy.

After approval of a novel drug such as T-VEC, standard operating procedures (SOPs) must be introduced into the patient care delivery system. Dr. David Ollila and colleagues at the University of North Carolina have described the implementation of such a T-VEC program at their cancer center. ${ }^{8}$ As stated in their article, "pre-implementation planning and clear designation of each team member's role can improve the quality of treatment and chance for remission for patients who suffer from advanced melanoma."

Finally, this series includes an important manuscript from the report of the MSLT I randomized melanoma surgical trial reported by Faries et al.. ${ }^{9}$ This article reminds us that there is a valuable, although palliative, role for surgical excision in select patients with stage IV melanoma, especially in the context of a well-designed, randomized clinical trial for advanced cancer, in which the role of surgical excision of isolated distant metastases may be critical for staging patients and even delaying relapses for many months or years. Given the expense of recently approved drug therapies, surgical resection is also likely to be relatively cost-effective in properly selected patients. ${ }^{9}$ Moreover, successful development of numerous drug therapies has changed the context in which resection of melanoma metastases is considered $;^{9}$ For example, far more patients might benefit from surgical resection to either consolidate their responses, stage the completeness of systemic treatment (i.e., determine whether the "mass" 
still has viable tumor cells), and allow performance of molecular analysis of treatment-refractory metastatic tumors that might require a completely different systemic treatment regimen after surgery.

\section{CLARION CALL: ARE SURGEONS PREPARED TO INCORPORATE THESE NEW AGENTS INTO THEIR ONCOLOGY PRACTICE?}

Never before in the history of cancer treatment has drug development advanced so rapidly for so many types of advanced cancer, which are now being evaluated in hundreds of clinical trials in surgical patients with cancers of many histologies. If surgeons are going to be full partners with their medical and radiation oncology colleagues in treatment planning and delivery of these new agents, we must incorporate these advances into our clinical knowledge, be informed participants in multidisciplinary treatment planning, and be active clinical investigators in clinical trials involving adjuvant and neoadjuvant systemic treatments. This is especially true for the use of neoadjuvant therapies with regard to the key decisions about the duration of systemic neoadjuvant treatment, the timing and extent of surgery to maximize local/regional disease control, and the provision of tissue suitable for molecular and genetic testing of the tumor to pathologists. If we are not engaged, surgeons may be relegated to a treatment position of "last resort" after systemic and radiation treatments have failed. The clarion call to all surgeons treating cancer is that we have vital roles to serve our patients as surgeons, as oncologists, and as clinical investigators.

In 1992, my Society of Surgical Oncology (SSO) presidential address included a prescient statement that still applies to our surgical oncology profession 25 years later. ${ }^{15}$ I stated then that "The increased emphasis on multidisciplinary cancer care raises the important question about which physician specialty will coordinate cancer care in the future... The traditional sequence of cancer management has involved surgery alone or surgery as the first of a series of multidisciplinary treatments with the surgeon being the patient's entry point into the care system because we establish the diagnosis and give the first treatment. However, by the $21^{\text {st }}$ century it is possible that only a minority of patients with cancer will have surgery alone as a single modality of treatment. It is more likely that chemotherapy and even radiation therapy will be used as the initial treatment for many patients; while surgical treatment for some types of cancer will be relegated to a secondary or even a tertiary level." 15

The opportunities for helping our patients are great in this rapidly evolving setting of incorporating new immunotherapy agents, targeted therapies, and molecular therapies into multidisciplinary cancer treatment planning with patient selection driven by biomarkers. We are entering a new epoch of cancer treatment with a vast portfolio of new and effective systemic agents. The availability of hundreds of agents alone or in combination presents a challenge for surgical oncologists, who must keep abreast of a rapidly changing landscape of treatment options.

The primary challenge going forward is to define the right drug combination and sequence in the right patients who are selected on the basis of their cancer's molecular/genetic signatures. The field is rapidly advancing, and we must keep up with these advances, participate in clinical trials that define new standards of care, and incorporate them into our standard practice. Are we ready?

ACKNOWLEDGMENTS Robert H.I. Andtbacka, MD, is acknowledged for his contributions in concept to the descriptive information about the genetically engineered oncolytic virus talimogene laherparepvec (T-VEC), and Gail Flores, $\mathrm{PhD}$, is thanked for her literature review and editorial contributions to this introduction/ editorial.

\section{REFERENCES}

1. Hodi FS, O’Day SJ, McDermott DF, et al. Improved survival with ipilimumab in patients with metastatic melanoma. $N$ Engl $J$ Med. 2010;363(8):711-723.

2. Ribas A, Hamid O, Daud A, et al. Association of pembrolizumab with tumor response and survival among patients with advanced melanoma. JAMA. 2016;315(15):1600-1609.

3. Callahan MK, Kluger H, Postow MA, et al. Nivolumab plus ipilimumab in patients with advanced melanoma: updated survival, response, and safety data in a phase I dose-escalation study. J Clin Oncol. 2018;36(4):391-398.

4. McDermott D, Lebbé C, Hodi FS, et al. Durable benefit and the potential for long-term survival with immunotherapy in advanced melanoma. Cancer Treat Rev. 2014;40(9):1056-1064.

5. Wolchok JD, Chiarion-Sileni V, Gonzalez R, et al. Overall survival with combined nivolumab and ipilimumab in advanced melanoma. N Engl J Med. 2017;377(14):1345-1356.

6. Bello DM, Ariyan CE. Adjuvant therapy in the treatment of melanoma. Ann Surg Oncol. 2018: https://doi.org/10.1245/s104 34-018-6376-y.

7. Keung EZ, Ukponmwan EU, Cogdill AP, Wargo JA. The rationale and emerging use of neoadjuvant immune checkpoint blockade in solid malignancies. Ann Surg Oncol. 2018: https://d oi.org/10.1245/s10434-018-6379-8.

8. Collichio F, Burke L, Proctor A, et al. Implementing a program of talimogene laherparepvec. Ann Surg Oncol. 2018: https://doi.org/ 10.1245/s10434-018-6361-6365.

9. Faries MB, Mozzillo N, Kashani-Sabet M, et al. Long-term survival after complete surgical resection and adjuvant immunotherapy for distant melanoma metastases. Ann Surg Oncol. 2017;24(13):3991--4000.

10. Andtbacka RHI, Kaufman KL, Collichio F, et al. Talimogene laherparepvec improves durable response rate in patients with advanced melanoma. J Clin Oncol. 2015;33(25):2770-2788.

11. Andtbacka RHI, Ross M, Puzanov I, et al. Patterns of clinical response with talimogene laherparepvec (T-VEC) in patients with 
melanoma treated in the OPTiM phase III clinical trial. Ann Surg Oncol. 2016;23(13):4169-4177.

12. IMLYGIC (talimogene laherparepvec) Prescribing Information. October 2015. Amgen Inc.: Thousand Oaks, CA.

13. Chesney J, Puzanov I, Collichio F, et al. Randomized, open-label phase II study evaluating the efficacy and safety of talimogene laherparepvec in combination with ipilimumab versus ipilimumab alone in patients with advanced, unresectable melanoma. J Clin Oncol. 2017;35. [Epub ahead of print].

14. Ribas A, Dummer R, Puzanov I, et al. Oncolytic virotherapy promotes intratumoral $\mathrm{T}$ cell infiltration and improves anti-PD-1 immunotherapy. Cell. 2017;170(6):1109-1119.

15. Balch CM. Presidential address: Surgical oncology in the $21 \mathrm{st}$ century. Arch Surg. 1992;127:1272-1277. 